\title{
Some Limit Properties of the Harmonic Mean of Transition Probabilities for Markov Chains in Markovian Environments Indexed by Cayley's Trees
}

\author{
Huilin Huang \\ College of Mathematics and Information Science, Wenzhou University, Zhejiang 325035, China \\ Correspondence should be addressed to Huilin Huang; huilin_huang@sjtu.org
}

Received 7 August 2013; Revised 24 October 2013; Accepted 31 October 2013

Academic Editor: Onesimo Hernandez Lerma

Copyright @ 2013 Huilin Huang. This is an open access article distributed under the Creative Commons Attribution License, which permits unrestricted use, distribution, and reproduction in any medium, provided the original work is properly cited.

We prove some limit properties of the harmonic mean of a random transition probability for finite Markov chains indexed by a homogeneous tree in a nonhomogeneous Markovian environment with finite state space. In particular, we extend the method to study the tree-indexed processes in deterministic environments to the case of random enviroments.

\section{Introduction}

A tree $T$ is a graph which is connected and doesn't contain any circuits. Given any two vertices $\alpha \neq \beta \in T$, let $\overline{\alpha \beta}$ be the unique path connecting $\alpha$ and $\beta$. Define the graph distance $d(\alpha, \beta)$ to be the number of edges contained in the path $\overline{\alpha \beta}$.

Let $T$ be an infinite tree with root 0 . The set of all vertices with distance $n$ from the root is called the $n$th generation of $T$, which is denoted by $L_{n}$. We denote by $T^{(n)}$ the union of the first $n$ generations of $T$. For each vertex $t$, there is a unique path from 0 to $t$ and $|t|$ for the number of edges on this path. We denote the first predecessor of $t$ by $1 t$. The degree of a vertex is defined to be the number of neighbors of it. If every vertex of the tree has degree $d+1$, we say it is Cayley's tree, which is denoted by $T_{C, d}$. Thus, the root vertex has $d+1$ neighbors in the first generation and every other vertex has $d$ neighbors in the next generation. For any two vertices $s$ and $t$ of tree $T$, write $s \leq t$ if $s$ is on the unique path from the root 0 to $t$. We denote by $s \wedge t$ the farthest vertex from 0 satisfying $s \wedge t \leq s$ and $s \wedge t \leq t$. We use the notation $X^{A}=\left\{X_{t}, t \in A\right\}$ and denote by $|A|$ the number of vertices of $A$.

In the following, we always let $T$ denote the Cayley tree $T_{C, d}$.
A tree-indexed Markov chain is the particular case of a Markov random field on a tree. Kemeny et al. [1] and Spitzer [2] introduced two special finite tree-indexed Markov chains with finite transition matrix which is assumed to be positive and reversible to its stationary distribution, and these treeindexed Markov chains ensure that the cylinder probabilities are independent of the direction we travel along a path. In this paper, we omit such assumption and adopt another version of the definition of tree-indexed Markov chains which is put forward by Benjamini and Peres [3]. Yang and Ye[4] extended it to the case of nonhomogeneous Markov chains indexed by infinite Cayley's tree and we restate it here as follows.

Definition 1 ( $T$-indexed nonhomogeneous Markov chains (see [4])). Let $T$ be an infinite Cayley tree, $\mathscr{X}$ a finite state space, and $\left\{X_{t}, t \in T\right\}$ a stochastic process defined on probability space $(\Omega, F, \mathbf{P})$, which takes values in the finite set $\mathscr{X}$. Let

$$
p=\{p(i), i \in \mathscr{X}\}
$$

be a distribution on $\mathscr{X}$ and

$$
P_{t}=\left(P_{t}(j \mid i)\right), \quad i, j \in \mathscr{X},
$$


a transition probability matrix on $\mathscr{X}^{2}$. If, for any vertex $t$,

$$
\begin{gathered}
\mathbf{P}\left(X_{t}=j \mid X_{1 t}=i, X_{s}=x_{s} \text { for } t \wedge s \leq 1 t\right) \\
=\mathbf{P}\left(X_{t}=j \mid X_{1 t}=i\right)=P_{t}(j \mid i), \quad \forall i, j \in \mathscr{X}, \\
\mathbf{P}\left(X_{0}=i\right)=p(i) \quad \forall i \in \mathscr{X},
\end{gathered}
$$

then $\left\{X_{t}, t \in T\right\}$ will be called $\mathscr{X}$-valued nonhomogeneous Markov chains indexed by infinite Cayley's tree with initial distribution (1) and transition probability matrices $\left\{P_{t}, t \in\right.$ $T\}$.

The subject of tree-indexed processes has been deeply studied and made abundant achievements. Benjamini and Peres [3] have given the notion of the tree-indexed Markov chains and studied the recurrence and ray-recurrence for them. Berger and Ye [5] have studied the existence of entropy rate for some stationary random fields on a homogeneous tree. Ye and Berger $[6,7]$, by using Pemantle's result [8] and a combinatorial approach, have studied the ShannonMcMillan theorem with convergence in probability for a PPG-invariant and ergodic random field on a homogeneous tree. Yang and Liu [9] and Yang [10] have studied a strong law of large numbers for Markov chains fields on a homogeneous tree (a particular case of tree-indexed Markov chains and PPG-invariant random fields). Yang and Ye [4] have established the Shannon-McMillan theorem for nonhomogeneous Markov chains on a homogeneous tree. Huang and Yang [11] has studied the strong law of large numbers for finite homogeneous Markov chains indexed by a uniformly bounded infinite tree.

The previous results are all about tree-indexed processes in deterministic environments. Recently, we are interested in random fields indexed by trees in random environments. In the rest of this paper we formulate a model of Markov chain indexed by trees in random environment especially in Markovian environment and study some limit properties of the harmonic mean of random transition probability for finite Markov chains indexed by a homogeneous tree in a nonhomogeneous Markovian environment. We are also interested in the strong law of large numbers of Markov chains indexed by trees in random environments which we will prepare for in another paper.

Definition 2. Let $T$ be an infinite Cayley tree, $\mathscr{X}$ and $\Theta$ two finite state spaces. Suppose that $\left\{\xi_{t}, t \in T\right\}$ is a $\Theta$-valued random field indexed by $T$, if, for any vertex $t \in T$,

$$
\begin{gathered}
\mathbf{P}\left(X_{t}=j \mid X_{1 t}=i, X_{s}=x_{s}, \text { for } t \wedge s \leq 1 t ; \xi_{r}, r \in T\right) \\
=P_{\xi_{1 t}}(i, j), \quad \text { a.s. } \\
\quad \mathbf{P}\left(X_{0}=i \mid \xi_{r}, r \in T\right)=P\left(i \mid \xi_{0}\right) \quad \forall i \in \mathscr{X}
\end{gathered}
$$

for each $i, j \in \mathscr{X}$, where $P_{x}=\left(P_{x}(i, j)\right)_{i, j \in \mathscr{X}}, x \in \Theta$ is a family of stochastic matrices. Then we call $\left\{X_{t}, t \in T\right\}$ a Markov chain indexed by tree $T$ in a random environment $\left\{\xi_{t}, t \in\right.$ $T\}$. The $\xi_{t}^{\prime} s$ are called the environmental process or control process indexed by tree $T$. Moreover, if $\left\{\xi_{t}, t \in T\right\}$ is a $T$ indexed Markov chain with initial distribution $\mu=\{\mu(\theta), \theta \in$
$\Theta\}$ and one-step transition probability matrices $K_{t}=$ $\left\{K_{t}(\alpha, \beta), \alpha, \beta \in \Theta, t \in T\right\}$, we call $\left\{X_{t}, t \in T\right\}$ a Markov chain indexed by tree $T$ in a nonhomogeneous Markovian environment.

Remark 3. If every vertex has degree two, then our model of Markov chain indexed by homogeneous tree with Markovian environments is reduced to the model of Markov chain in Markovian environments which was introduced by Cogburn [12] in spirit.

Remark 4. We also point out that our model is different from the tree-valued random walk in random environment (RWRE) that is studied by Pemantle and Peres [13] and $\mathrm{Hu}$ and Shi [14]. For the model of RWRE on the trees that they studied, the random environment process $\omega$ := $(\omega(x, \cdot), x, y \in T)$ is a family of i.i.d nondegenerate random vectors and the process $X=\left\{X_{n}, n \in \mathbf{Z}^{+}\right\}$is a nearest-neighbor walk satisfying some conditions. But in our model,our environmental process $\xi=\left\{\xi_{t}, t \in T\right\}$ can be any Markov chain indexed by trees. Given $\xi$, the process $\left\{X_{t}, t \in\right.$ $T\}$ is another Markov chain indexed by trees with the law $P_{\xi}$.

In this paper we assume that $\left\{\xi_{t}, t \in T\right\}$ is a nonhomogeneous $T$-indexed Markov chain on state space $\Theta$. The probability of going from $i$ to $j$ in one step in the $\theta$ th environment is denoted by $P_{\theta}(i, j)$. We also suppose that the one-step transition probability of going from $\alpha$ to $\beta$ for nonhomogeneous $T$-indexed Markov chain $\left\{\xi_{t}, t \in T\right\}$ is $K_{t}(\alpha, \beta)$. In this case, $\left\{\xi_{t}, X_{t}, t \in T\right\}$ is a Markov chain indexed by $T$ with initial distribution $q=$ $(q(\theta, i))$ and one-step transition on $\Theta \times \mathscr{X}$ determined by

$$
P_{t}(\alpha, i ; \beta, j)=K_{t}(\alpha, \beta) P_{\alpha}(i, j),
$$

where $q(\theta, i)=\mathbf{P}\left(\xi_{0}=\theta, X_{0}=i\right)$. Then $\left\{\xi_{t}, X_{t}, t \in T\right\}$ will be called the bichain indexed by tree $T$. Obviously, we have

$$
\begin{aligned}
& \mathbf{P}\left(\xi^{T^{(n)}}=\alpha^{T^{(n)}}, X^{T^{(n)}}=x^{T^{(n)}}\right) \\
& \quad=q\left(\alpha_{0}, x_{0}\right) \prod_{t \in T^{(n)}\{\{0\}} P_{t}\left(\alpha_{1 t}, x_{1 t} ; \alpha_{t}, x_{t}\right) .
\end{aligned}
$$

\section{Main Results}

For every finite $n \in \mathbf{N}$, let $\left\{X_{t}, t \in T\right\}$ be a Markov chain indexed by an infinite Cayley tree $T$ in Markovian environment $\left\{\xi_{t}, t \in T\right\}$, which is defined as in Definition 2. Now we suppose that $g_{t}(\alpha, i, \beta, j)$ are functions defined on $\Theta \times \mathscr{X} \times \Theta \times \mathscr{X}$. Let $\lambda$ be a real number, $L_{0}=\{0\}, \mathscr{F}_{n}=$ $\sigma\left(\xi^{T^{(n)}}, X^{T^{(n)}}\right)$; now we define a stochastic sequence as follows:

$$
\varphi_{n}(\lambda, \omega)=\frac{e^{\lambda \sum_{t \in T^{(n)}\{\{0\}} g_{t}\left(\xi_{1}, X_{1 t}, \xi_{t}, X_{t}\right)}}{\prod_{t \in T^{(n)} \backslash\{0\}} E\left[e^{\lambda g_{t}\left(\xi_{1 t}, X_{1 t}, \xi_{t}, X_{t}\right)} \mid \xi_{1 t}, X_{1 t}\right]} .
$$

At first we come to prove the following fact.

Lemma 5. $\left\{\varphi_{n}(\lambda, \omega), \mathscr{F}_{n}, n \geq 1\right\}$ is a nonnegative martingale. 
Proof of Lemma 5. Obviously, we have

$$
\begin{aligned}
\mathbf{P} & \left(\xi^{L_{n}}=\alpha^{L_{n}}, X^{L_{n}}=x^{L_{n}} \mid \xi^{T^{(n-1)}}\right. \\
& \left.=\alpha^{T^{(n-1)}}, X^{T^{(n-1)}}=x^{T^{(n-1)}}\right) \\
& =\frac{\mathbf{P}\left(\xi^{T^{(n)}}=\alpha^{T^{(n)}}, X^{T^{(n)}}=x^{T^{(n)}}\right)}{\mathbf{P}\left(\xi^{T^{(n-1)}}=\alpha^{T^{(n-1)}}, X^{T^{(n-1)}}=x^{\left.T^{(n-1)}\right)}\right.} \\
& =\prod_{t \in L_{n}} \mathbf{P}\left(\xi_{t}=\alpha_{t}, X_{t}=x_{t} \mid \xi_{1 t}=\alpha_{1 t}, X_{1 t}=x_{1 t}\right) .
\end{aligned}
$$

Here, the second equation holds because of the fact that $\left\{\xi_{t}, X_{t}, t \in T\right\}$ is a bichain indexed by tree $T$ and (8) is being used. Furthermore, we have

$$
\begin{aligned}
E[ & \left.e^{\lambda \sum_{t \in L_{n}} g_{t}\left(\xi_{1 t}, X_{1 t}, \xi_{t}, X_{t}\right)} \mid \mathscr{F}_{n-1}\right] \\
= & \sum_{\alpha^{L_{n}}, x^{L_{n}}} e^{\lambda \sum_{t \in L_{n}} g_{t}\left(\xi_{1 t}, X_{1 t}, \alpha_{t}, x_{t}\right)} \\
& \times \mathbf{P}\left(\xi^{L_{n}}=\alpha^{L_{n}}, X^{L_{n}}=x^{L_{n}} \mid \xi^{T^{(n-1)}}, X^{T^{(n-1)}}\right) \\
= & \sum_{\alpha^{L_{n}}, x^{L_{n}}} \prod_{t \in L_{n}} e^{\lambda g_{t}\left(\xi_{1 t}, X_{1 t}, \alpha_{t}, x_{t}\right)} \\
= & \left.\prod_{t \in L_{n}} \times \mathbf{P}\left(\xi_{t}, \xi_{t}\right) \alpha_{t}, X_{t}=x_{t} \mid \xi_{1 t}, X_{1 t}\right) \\
& \times \mathbf{P}\left(\xi_{t}=\alpha_{t}, X_{t}=x_{t} \mid \xi_{1 t}, X_{1 t}\right) \\
= & \prod_{t \in L_{n}} E\left[e^{\lambda g_{t}\left(\xi_{1 t}, X_{1 t}, \xi_{t}, X_{t}\right)} \mid \xi_{1 t}, X_{1 t}\right] \quad \text { a.s. }
\end{aligned}
$$

On the other hand, we also have

$$
\begin{aligned}
\varphi_{n}(\lambda, \omega)= & \varphi_{n-1}(\lambda, \omega) \\
& \times \frac{e^{\lambda \sum_{t \in L_{n}} g_{t}\left(\xi_{1 t}, X_{1 t}, \xi_{t}, X_{t}\right)}}{\prod_{t \in L_{n}} E\left[e^{\lambda g_{t}\left(\xi_{1 t}, X_{1 t}, \xi_{t}, X_{t}\right)} \mid \xi_{1 t}, X_{1 t}\right]}
\end{aligned}
$$

Combining (11) and (12), we get

$$
E\left[\varphi_{n}(\lambda, \omega) \mid \mathscr{F}_{n-1}\right]=\varphi_{n-1}(\lambda, \omega) \text { a.s. }
$$

Thus, we complete the proof of Lemma 5.

Theorem 6. Let $\left\{X_{t}, t \in T\right\}$ be a Markov chain indexed by an infinite Cayley tree $T$ in a nonhomogeneous Markovian environment $\left\{\xi_{t}, t \in T\right\}$. Suppose that the initial distribution and the transition probability functions satisfy

$$
\begin{gathered}
q\left(\alpha_{0}, x_{0}\right)>0, \quad P_{t}(\alpha, i ; \theta, j)>0, \\
\text { for } \forall \alpha_{0}, \alpha, \theta \in \Theta ; x_{0}, i, j \in \mathscr{X}, \\
a_{t}=\min \left\{P_{t}(\alpha, i ; \theta, j), \alpha, \theta \in \Theta ; i, j \in \mathscr{X}\right\}, t \in T \backslash\{0\},
\end{gathered}
$$

if there exist two positive constants $c$ and $m$ such that

$$
\limsup _{n \rightarrow \infty} \frac{1}{\left|T^{(n)}\right|} \sum_{t \in T^{(n)} \backslash\{0\}} e^{c / a_{t}}=m<\infty .
$$

Denote $|\Theta|=M,|\mathscr{X}|=N$, and

$$
P_{t}\left(\xi_{1 t}, X_{1 t} ; \xi_{t}, X_{t}\right)=P_{t}\left(\xi_{t}, X_{t} \mid \xi_{1 t}, X_{1 t}\right)
$$

then we have

$$
\lim _{n \rightarrow \infty} \frac{\left|T^{(n)}\right|}{\sum_{t \in T^{(n)} \backslash\{0\}} P_{t}\left(\xi_{1 t}, X_{1 t} ; \xi_{t}, X_{t}\right)^{-1}}=\frac{1}{M N} \quad \text { a.s. }
$$

Proof. By Lemma 5, we have known that $\left\{\varphi_{n}(\lambda, \omega), \mathscr{F}_{n}, n \geq\right.$ $1\}$ is a nonnegative martingale. According to Doob martingale convergence theorem, we have

$$
\lim _{n} \varphi_{n}(\lambda, \omega)=\varphi(\lambda, \omega)<\infty \quad \text { a.s. }
$$

so that

$$
\lim _{n \rightarrow \infty} \frac{\ln \varphi_{n}(\lambda, \omega)}{\left|T^{(n)}\right|}=0 \quad \text { a.s., }
$$

which implies that

$$
\limsup _{n \rightarrow \infty} \frac{\ln \varphi_{n}(\lambda, \omega)}{\left|T^{(n)}\right|} \leq 0 \quad \text { a.s. }
$$

We arrive at

$$
\begin{aligned}
& \limsup _{n \rightarrow \infty} \frac{1}{\left|T^{(n)}\right|} \\
& \times \sum_{t \in T^{(n)} \backslash\{0\}}\left\{\lambda g_{t}\left(\xi_{1 t}, X_{1 t}, \xi_{t}, X_{t}\right)\right. \\
&\left.\quad-\ln \left[E\left[e^{\lambda g_{t}\left(\xi_{1 t}, X_{1 t}, \xi_{t}, X_{t}\right)} \mid \xi_{1 t}, X_{1 t}\right]\right]\right\} \leq 0 \quad \text { a.s. }
\end{aligned}
$$

Combining (20) with the inequalities $\ln x \leq x-1$ $(x>0)$ and $0 \leq e^{x}-1-x \leq 2^{-1} x^{2} e^{|x|}$ and taking $g_{t}\left(\xi_{1 t}, X_{1 t}, \xi_{t}, X_{t}\right)=P_{t}\left(\xi_{1 t}, X_{1 t} ; \xi_{t}, X_{t}\right)^{-1}$, it follows that

$$
\begin{aligned}
& \limsup _{n \rightarrow \infty} \frac{1}{\left|T^{(n)}\right|} \sum_{t \in T^{(n)} \backslash\{0\}}\left[P_{t}\left(\xi_{1 t}, X_{1 t} ; \xi_{t}, X_{t}\right)^{-1} \lambda-\lambda M N\right] \\
& \leq \limsup _{n \rightarrow \infty} \frac{1}{\left|T^{(n)}\right|} \\
& \quad \times \sum_{t \in T^{(n)} \backslash\{0\}}\left\{\ln \left[E\left[e^{\lambda P_{t}\left(\xi_{1 t}, X_{1 t} ; \xi_{t}, X_{t}\right)^{-1}} \mid \xi_{1 t}, X_{1 t}\right]\right]\right. \\
& -\lambda M N\}
\end{aligned}
$$




$$
\begin{aligned}
& \leq \limsup _{n \rightarrow \infty} \frac{1}{\left|T^{(n)}\right|} \\
& \times \sum_{t \in T^{(n)} \backslash\{0\}}\left\{E\left[e^{\lambda P_{t}\left(\xi_{1 t}, X_{1 t} ; \xi_{t}, X_{t}\right)^{-1}} \mid \xi_{1 t}, X_{1 t}\right]\right. \\
& \quad-1-\lambda M N\} \\
& \leq \limsup _{n \rightarrow \infty} \frac{1}{\left|T^{(n)}\right|} \\
& \quad \times \sum_{t \in T^{(n)} \backslash\{0\}} \sum_{\alpha \in \Theta} \sum_{i \in \mathscr{X}} P_{t}\left(\xi_{1 t}, X_{1 t} ; \alpha, i\right) \\
& \left.\quad \times e^{\lambda / P_{t}\left(\xi_{1 t}, X_{1 t} ; \alpha, i\right)}-1-\frac{\lambda}{P_{t}\left(\xi_{1 t}, X_{1 t} ; \alpha, i\right)}\right] \\
& \leq \frac{\lambda^{2}}{2} \limsup _{n \rightarrow \infty} \frac{1}{\left|T^{(n)}\right|} \\
& \quad \times \sum_{t \in T^{(n)} \backslash\{0\}} \sum_{\alpha \in \Theta} \sum_{i \in \mathscr{X}} \frac{1}{P_{t}\left(\xi_{1 t}, X_{1 t} ; \alpha, i\right)} e^{|\lambda| / P_{t}\left(\xi_{1 t}, X_{1 t} ; \alpha, i\right)} \\
& \leq \frac{\lambda^{2} M N}{2} \limsup _{n \rightarrow \infty} \frac{1}{\left|T^{(n)}\right|} \sum_{t \in T^{(n)} \backslash\{0\}} \frac{1}{a_{t}} e^{|\lambda| / a_{t}} \text { a.s. }
\end{aligned}
$$

Note that the following elementary fact holds:

$$
\max _{x>0}\left\{x \gamma^{x}\right\}=-\frac{e^{-1}}{\ln \gamma}, \quad 0<\gamma<1 .
$$

Let $0<\lambda<c$. It follows from (15), (22), and (23) that

$$
\begin{aligned}
& \limsup _{n \rightarrow \infty} \frac{1}{\left|T^{(n)}\right|} \sum_{t \in T^{(n)} \backslash\{0\}}\left[P_{t}\left(\xi_{1 t}, X_{1 t} ; \xi_{t}, X_{t}\right)^{-1}-M N\right] \\
& \quad \leq \frac{\lambda M N}{2} \limsup _{n \rightarrow \infty} \frac{1}{\left|T^{(n)}\right|} \sum_{t \in T^{(n)} \backslash\{0\}} \frac{1}{a_{t}} e^{\lambda / a_{t}} \\
& \quad=\frac{\lambda M N}{2} \limsup _{n \rightarrow \infty} \frac{1}{\left|T^{(n)}\right|} \sum_{t \in T^{(n)} \backslash\{0\}} \frac{1}{a_{t}}\left(e^{\lambda-c}\right)^{1 / a_{t}} e^{c / a_{t}} \\
& \quad \leq \frac{\lambda M N}{2 e(c-\lambda)} m \text { a.s. }
\end{aligned}
$$

Here, the second equation holds because by letting $\lambda \rightarrow$ $0^{+}$in inequality (24), we get

$$
\begin{aligned}
\limsup _{n \rightarrow \infty} & \frac{1}{\left|T^{(n)}\right|} \\
& \times \sum_{t \in T^{(n)} \backslash\{0\}}\left[P_{t}\left(\xi_{1 t}, X_{1 t} ; \xi_{t}, X_{t}\right)^{-1}-M N\right] \leq 0 \quad \text { a.s. }
\end{aligned}
$$

If $-c<\lambda<0$, similar to the analysis of inequality (24), by using (15), (22), and (23) again, we can arrive at

$$
\begin{aligned}
& \liminf _{n \rightarrow \infty} \frac{1}{\left|T^{(n)}\right|} \sum_{t \in T^{(n)} \backslash\{0\}}\left[P_{t}\left(\xi_{1 t}, X_{1 t} ; \xi_{t}, X_{t}\right)^{-1}-M N\right] \\
& \geq \frac{\lambda M N}{2} \limsup _{n \rightarrow \infty} \frac{1}{\left|T^{(n)}\right|} \sum_{t \in T^{(n)} \backslash\{0\}} \frac{1}{a_{t}} e^{-\lambda / a_{t}} \\
& \geq \frac{\lambda M N}{2} \limsup _{n \rightarrow \infty} \frac{1}{\left|T^{(n)}\right|} \sum_{t \in T^{(n)} \backslash\{0\}} \frac{1}{a_{t}}\left(\frac{e^{-\lambda}}{e^{c}}\right)^{1 / a_{t}} e^{c / a_{t}} \\
& \geq \frac{\lambda M N}{2 e(\lambda+c)} m \text { a.s. }
\end{aligned}
$$

Letting $\lambda \rightarrow 0^{-}$in inequality (26), we get

$$
\begin{aligned}
\liminf _{n \rightarrow \infty} & \frac{1}{\left|T^{(n)}\right|} \\
& \times \sum_{t \in T^{(n)} \backslash\{0\}}\left[P_{t}\left(\xi_{1 t}, X_{1 t} ; \xi_{t}, X_{t}\right)^{-1}-M N\right] \geq 0 \quad \text { a.s. }
\end{aligned}
$$

Combining (25) and (27), we obtain that our assertion (17) is true.

Corollary 7. Let $\left\{X_{t}, t \in T\right\}$ be a nonhomogeneous Markov chain indexed by an infinite Cayley tree T. Suppose that the initial distribution and the transition probability functions satisfy

$$
\begin{gathered}
p\left(x_{0}\right)>0, \quad P_{t}(i, j)>0, \\
a_{t}=\min \left\{P_{t}(i, j), i, j \in \mathscr{X}\right\}, \quad t \in T \backslash\{0\},
\end{gathered}
$$

if there exist two positive constants $c$ and $m$ such that

$$
\limsup _{n \rightarrow \infty} \frac{1}{\left|T^{(n)}\right|} \sum_{t \in T^{(n)} \backslash\{0\}} e^{c / a_{t}}=m<\infty .
$$

Denote $|\mathscr{X}|=N$; then we have

$$
\lim _{n \rightarrow \infty} \frac{\left|T^{(n)}\right|}{\sum_{t \in T^{(n)} \backslash\{0\}} P_{t}\left(X_{t} \mid X_{1 t}\right)^{-1}}=\frac{1}{N} \quad \text { a.s. }
$$

Proof. If we take $\Theta=\{\theta\}$, that is, $|\Theta|=1$, then the model of Markov chain indexed by tree $T$ in Markovian environment reduces to the formulation of a nonhomogeneous Markov chain indexed by tree $T$. Then we arrive at our conclusion (30) directly from Theorem 6.

Corollary 8 (see [15]). Let $\left\{X_{n}, n \geq 0\right\}$ be a Markov chain in a nonhomogeneous Markovian environment $\left\{\xi_{n}, n \geq 0\right\}$. Suppose that the initial distribution and the transition probability functions satisfy

$$
\begin{gathered}
q\left(\alpha_{0}, x_{0}\right)>0, \quad P_{n}(\alpha, i ; \theta, j)>0, \\
a_{n}=\min \left\{P_{n}(\alpha, i ; \theta, j), \alpha, \theta \in \Theta ; i, j \in \mathscr{X}\right\}, \quad n \geq 1,
\end{gathered}
$$


if there exist two positive constants $c$ and $m$ such that

$$
\limsup _{n \rightarrow \infty} \frac{1}{n} \sum_{k=1}^{n} e^{c / a_{k}}=m<\infty .
$$

Denote $|\Theta|=M,|\mathscr{X}|=N$, and

$$
P_{k}\left(\xi_{k-1}, X_{k-1} ; \xi_{k}, X_{k}\right)=P_{k}\left(\xi_{k}, X_{k} \mid \xi_{k-1}, X_{k-1}\right) ;
$$

then we have

$$
\lim _{n \rightarrow \infty} \frac{n}{\sum_{k=1}^{n} P_{k}\left(\xi_{k-1}, X_{k-1} ; \xi_{k}, X_{k}\right)^{-1}}=\frac{1}{M N} \quad \text { a.s. }
$$

Proof. If every vertex of the tree $T$ has degree 2, then the nonhomogeneous Markov chain indexed by tree $T$ degenerates into the nonhomogeneous Markov chain on line; thus, this corollary can be obtained from Theorem 6 directly.

\section{Acknowledgment}

This work was supported by the National Natural Science Foundation of China no. 11201344. The author declares that there is no conflict of interests regarding the publication of this paper.

\section{References}

[1] J. G. Kemeny, J. L. Snell, and A. W. Knapp, Denumerable Markov Chains, Springer, New York, NY, USA, 2nd edition, 1976.

[2] F. Spitzer, "Markov random fields on an infinite tree," The Annals of Probability, vol. 3, no. 3, pp. 387-398, 1975.

[3] I. Benjamini and Y. Peres, "Markov chains indexed by trees," The Annals of Probability, vol. 22, no. 1, pp. 219-243, 1994.

[4] W. Yang and Z. Ye, "The asymptotic equipartition property for nonhomogeneous Markov chains indexed by a homogeneous tree," IEEE Transactions on Information Theory, vol. 53, no. 9, pp. 3275-3280, 2007.

[5] T. Berger and Z. X. Ye, "Entropic aspects of random fields on trees," IEEE Transactions on Information Theory, vol. 36, no. 5, pp. 1006-1018, 1990.

[6] Z. Ye and T. Berger, "Ergodicity, regularity and asymptotic equipartition property of random fields on trees," Journal of Combinatorics, Information \& System Sciences, vol. 21, no. 2, pp. 157-184, 1996.

[7] Z. Ye and T. Berger, Information Measures for Discrete Random Fields, Science Press, Beijing, China, 1998.

[8] R. Pemantle, "Automorphism invariant measures on trees," The Annals of Probability, vol. 20, no. 3, pp. 1549-1566, 1992.

[9] W. Yang and W. Liu, "Strong law of large numbers for Markov chains field on a Bethe tree," Statistics \& Probability Letters, vol. 49, no. 3, pp. 245-250, 2000.

[10] W. Yang, "Some limit properties for Markov chains indexed by a homogeneous tree," Statistics \& Probability Letters, vol. 65, no. 3, pp. 241-250, 2003.

[11] H. Huang and W. Yang, "Strong law of large numbers for Markov chains indexed by an infinite tree with uniformly bounded degree," Science in China A, vol. 51, no. 2, pp. 195-202, 2008.
[12] R. Cogburn, "Markov chains in random environments: the case of Markovian environments," The Annals of Probability, vol. 8, no. 5, pp. 908-916, 1980.

[13] R. Pemantle and Y. Peres, "Critical random walk in random environment on trees," The Annals of Probability, vol. 23, no. 1, pp. 105-140, 1995.

[14] Y. Hu and Z. Shi, "Slow movement of random walk in random environment on a regular tree," The Annals of Probability, vol. 35, no. 5, pp. 1978-1997, 2007.

[15] Y. Q. Li, H. S. Wang, and Z. Wang, "Some strong limit theorems of geometric average of transition probabilities and their function for Markov chains in Markovian environments," Acta Mathematica Scientia A, vol. 31, no. 2, pp. 508-517, 2011 (Chinese). 


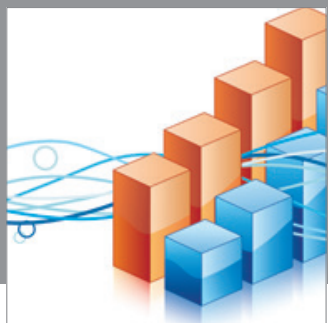

Advances in

Operations Research

mansans

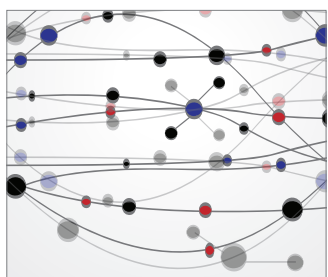

The Scientific World Journal
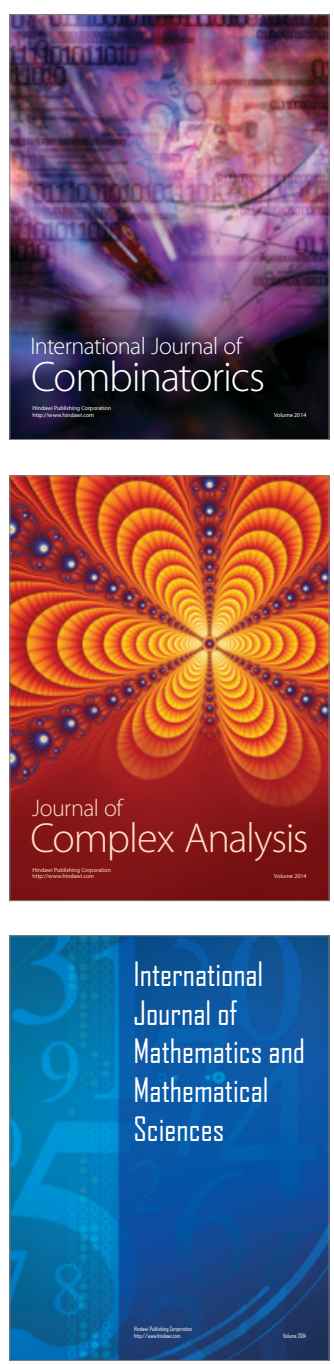
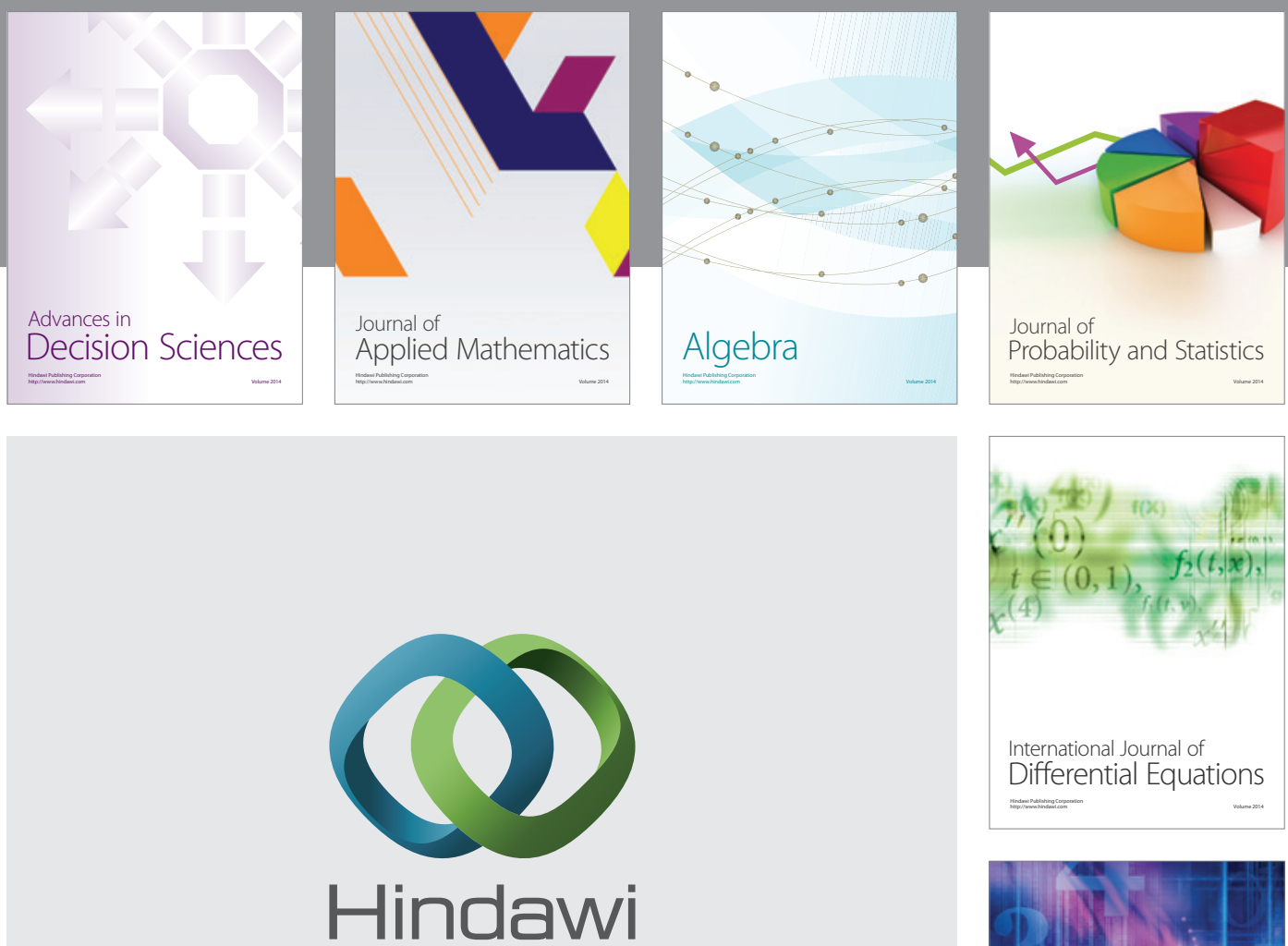

Submit your manuscripts at http://www.hindawi.com
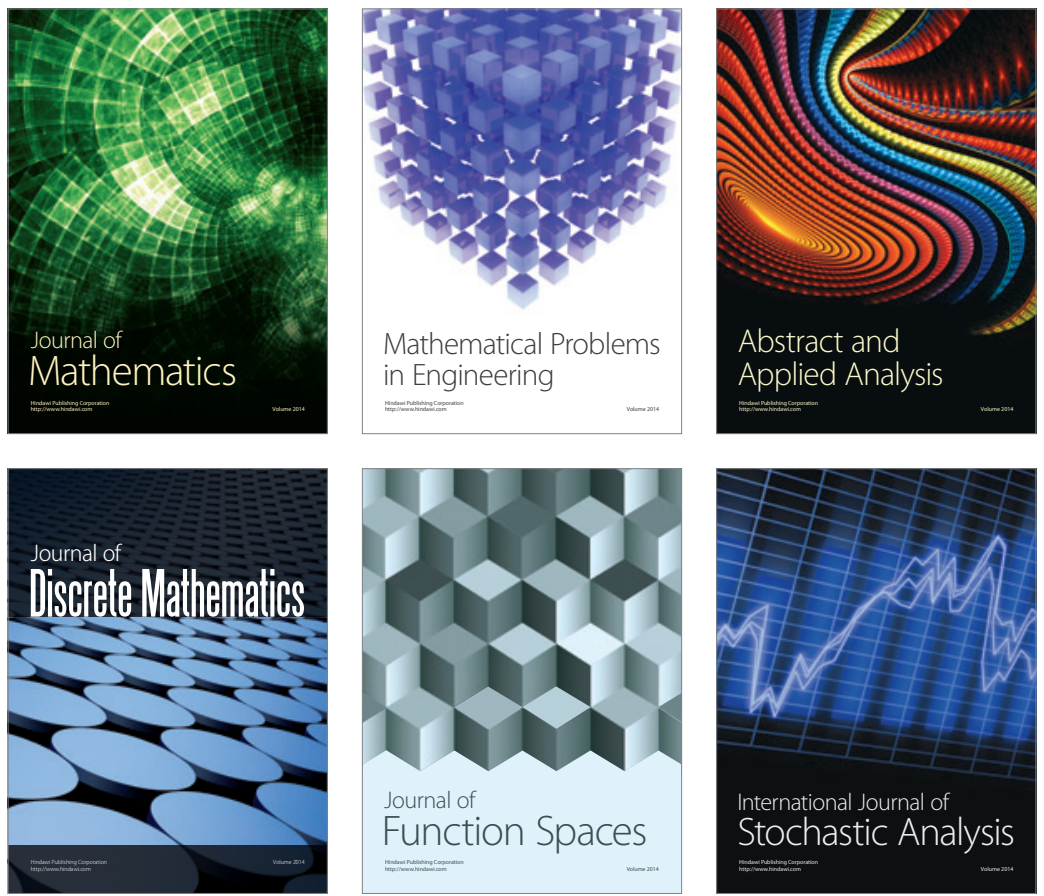

Journal of

Function Spaces

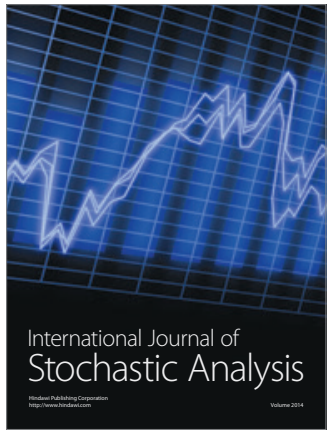

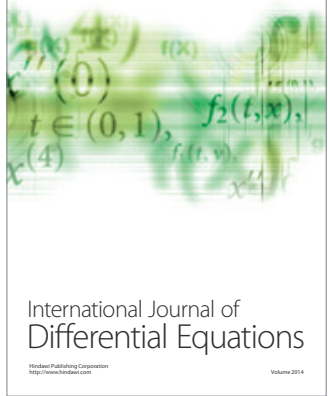
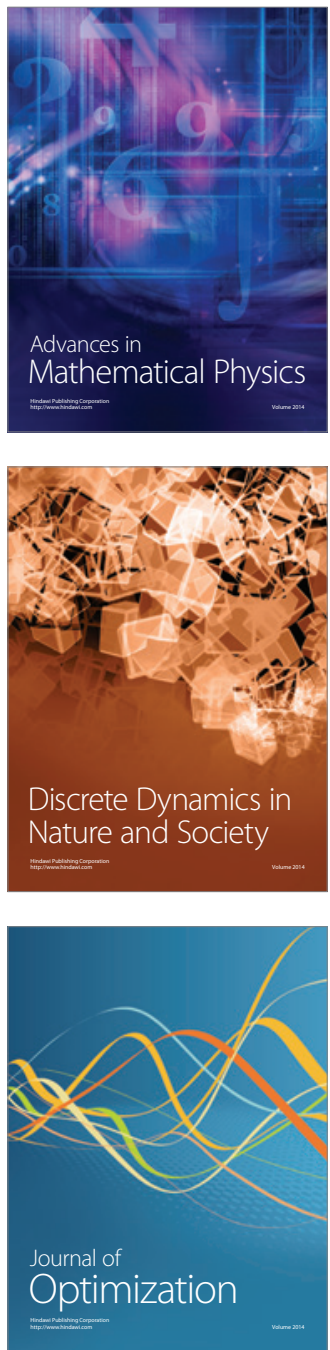